\title{
Heterologous production of raspberry ketone in the wine yeast Saccharomyces cerevisiae via pathway engineering and synthetic enzyme fusion
}

\author{
Danna Lee ${ }^{1}$, Natoiya D. R. Lloyd ${ }^{1}$, Isak S. Pretorius ${ }^{2}$ and Anthony R. Borneman $1,3^{*}$
}

\begin{abstract}
Background: Raspberry ketone is the primary aroma compound found in raspberries and naturally derived raspberry ketone is a valuable flavoring agent. The economic incentives for the production of raspberry ketone, combined with the very poor yields from plant tissue, therefore make this compound an excellent target for heterologous production in synthetically engineered microbial strains.
\end{abstract}

Methods: A de novo pathway for the production of raspberry ketone was assembled using four heterologous genes, encoding phenylalanine/tyrosine ammonia lyase, cinnamate-4-hydroxlase, coumarate-CoA ligase and benzalacetone synthase, in an industrial strain of Saccharomyces cerevisiae. Synthetic protein fusions were also explored as a means of increasing yields of the final product.

Results: The highest raspberry ketone concentration achieved in minimal media exceeded $7.5 \mathrm{mg} / \mathrm{L}$ when strains were fed with $3 \mathrm{mM}$ p-coumaric acid; or $2.8 \mathrm{mg} / \mathrm{L}$ for complete de novo synthesis, both of which utilized a coumarate-CoA ligase, benzalacetone synthase synthetic fusion protein that increased yields over fivefold compared to the native enzymes. In addition, this strain was shown to be able to produce significant amounts of raspberry ketone in wine, with a raspberry ketone titer of $3.5 \mathrm{mg} / \mathrm{L}$ achieved after aerobic fermentation of Chardonnay juice or $0.68 \mathrm{mg} / \mathrm{L}$ under anaerobic winemaking conditions.

Conclusions: We have shown that it is possible to produce sensorially-relevant quantities of raspberry ketone in an industrial heterologous host. This paves the way for further pathway optimization to provide an economical alternative to raspberry ketone derived from plant sources.

Keywords: Phenylpropanoid, Raspberry ketone, Synthetic engineering, Wine yeast

\section{Background}

Plant natural compounds represent a large, chemicallydiverse collection of secondary metabolites, however this diversity is generated from a limited number of conserved pathways (reviewed in Marienhagen et al. [1]). One such class of plant compounds are the phenylpropanoids, which like flavonoids, stilbenes and lignans, are formed

\footnotetext{
*Correspondence: anthony.borneman@awri.com.au

${ }^{3}$ Department of Genetics and Evolution, University of Adelaide, Adelaide,

SA 5000, Australia

Full list of author information is available at the end of the article
}

from the common metabolic precursor p-coumaric acid via the amino acids phenylalanine and tyrosine.

Raspberry ketone [4-(4-hydroxyphenyl)butan-2-one] is a phenylpropanoid that is found in many fruits, berries and vegetables, including raspberries, blackberries, grapes and rhubarb. As the name suggests, it is considered a major impact sensory molecule in raspberry, along with other volatile aroma compounds such as monoterpenes, $\beta$-damascenone and $\alpha$ - and $\beta$-ionone $[2$, 3]. Naturally-derived raspberry ketone is a valuable flavoring agent (US\$3000/kg), as plant-derived yields of this compound are very low, even from raspberries $(1-4 \mathrm{mg} /$ 
$\mathrm{kg})[3,4]$. Raspberry ketone can also be derived through chemical means, however this synthetic compound attracts a far lower price (US\$58/kg) than the naturallyderived form [4].

The economic incentives for the production of raspberry ketone, combined with the very poor yields from plant tissue, therefore make this compound an excellent target for production via the use of synthetically engineered microbial strains. Previous work has shown that it is possible to produce raspberry ketone from p-coumaric acid in heterologous systems such as Escherichia coli $(5 \mathrm{mg} / \mathrm{L})$ and $S$. cerevisiae (trace amounts) [5]. However, de novo production of raspberry ketone, without the need for precursor addition, has not yet been demonstrated.

In order to establish a heterologous system for raspberry ketone production, a de novo biosynthetic route, comprising four separate enzymatic activities, has been engineered into S. cerevisiae. Furthermore in order to optimize the production of this valuable aroma compound, synthetic protein fusions were explored and found to increase final concentrations of raspberry ketone over fivefold. Finally, as the metabolic engineering was performed in a wine strain of S. cerevisiae, we show that this engineered strain is capable of synthesizing raspberry ketone at concentrations almost two orders of magnitude above its predicted sensory threshold in Chardonnay grape juice under standard winemaking conditions, while retaining the ability to complete fermentation.

\section{Results and discussion}

\section{Biosynthesis of raspberry ketone from p-coumaric acid}

The production of raspberry ketone from $p$-coumaric acid requires the action of two heterologous enzyme activities, a coumarate-CoA ligase (4CL) and benzalacetone synthase (BAS), as yeast has been shown to natively display efficient benzalacetone reductase (BAR) activity [5] (Fig. 1a).

Based upon previous literature, two different 4CL genes were chosen for investigation, $A$. thaliana (At4CL1, GenBank: AAA82888.1) and parsley (Petroselinum crispum; Pc4CL2, GenBank: CAA31697.1) [6, 7]. For BAS activity, the rhubarb (Rheum palmatum) BAS (RpBAS, GenBank: AAK82824.1) carrying a S338 V mutation was selected, as this enzyme provides high BAS activity without the co-production of naringenin $[8,9]$.

Codon optimized versions of the heterologous genes were synthesized and the two different $4 \mathrm{CL}$ and BAS pairs (At4CL1 RpBAS and Pc4CL2 RpBAS) were integrated in the $H O$ locus of AWRI2975 (TDH3p:ACC1) in a tandem arrangement, with each gene driven from a separate copy of the highly-fermentation expressed promoter of $F B A 1[10,11]$ (Fig. 1b). The $H O$ locus was chosen as integration at this genomic location has been shown to be phenotypically neutral $[12,13]$. Fermentations, supplemented with p-coumaric acid ( $3 \mathrm{mM}$ final concentration), were performed with these two strains, with raspberry ketone levels of $0.37 \pm 0.01 \mathrm{mg} / \mathrm{L}$ and $0.43 \pm 0.01 \mathrm{mg} / \mathrm{L}$ observed for the At4CL1 RpBAS and Pc4CL2 RpBAS constructs, respectively (Fig. 2). While relatively low, these levels are nevertheless $>30-300$ fold above established sensory thresholds for raspberry ketone in aqueous solutions $(0.001-0.01 \mathrm{mg} / \mathrm{L})[2,14]$.

In order to attempt to increase the levels of raspberry ketone produced, a synthetic fusion enzyme strategy was employed, as this has been shown to increase yields of other heterologous metabolites, presumably through substrate funneling $[15,16]$. Short protein linkers that are predicted to have either a flexible (VDGGSGR) or rigid (VDEAAAKSGR) conformation [16] were used to fuse the coding regions of the $4 \mathrm{CL}$ and BAS enzymes in both tandem orientations (4CL-RpBAS and $R p$ BAS4CL), using either the $A$. thaliana or P. crispum 4CL genes (Fig. 1b). The production of raspberry ketone was then assessed for these four different constructs compared to the binary gene systems (Fig. 2). While the type of linker (flexible or rigid) did not significantly affect the observed levels of raspberry ketone, fusing the two genes in the $4 \mathrm{CL}-\mathrm{R} p$ BAS orientation provided 3.5- and 6.5fold increases in the levels of raspberry ketone produced with the A. thaliana $(1.29 \pm 0.09 \mathrm{mg} / \mathrm{L})$ or $P$. crispum $(2.81 \pm 1.38 \mathrm{mg} / \mathrm{L}) 4 \mathrm{CL}$ genes, respectively. However this effect was specific to the orientation of the fusion protein, as the $R p B A S-4 C L$ fusions provided no significant difference in raspberry ketone levels compared to the two independent genes.

\section{Effect of oxygen on the production of raspberry ketone}

As the Pc4CL2-RpBAS fusion constructs were shown to have the highest activity in anaerobic fermentation, the Pc4CL2-r-RpBAS strain was evaluated for raspberry ketone production under aerobic growth conditions, so that the effect of oxygen could be assessed (Fig. 3). The aerobic fermentation resulted in a significant increase in raspberry ketone production of 2.6-fold $(7.54 \pm 0.42 \mathrm{mg} / \mathrm{L})$.

\section{De novo biosynthesis of raspberry ketone}

While the aforementioned strains are capable of producing significant levels of raspberry ketone, they require the exogenous addition of the pathway precursor p-coumaric acid. In order to engineer the de novo production p-coumaric acid in S. cerevisiae from the aromatic amino acid precursors phenylalanine and tyrosine, three enzyme activities were required (Fig. 1), phenylalanine ammonia lyase (PAL), tyrosine ammonia 


\section{a}

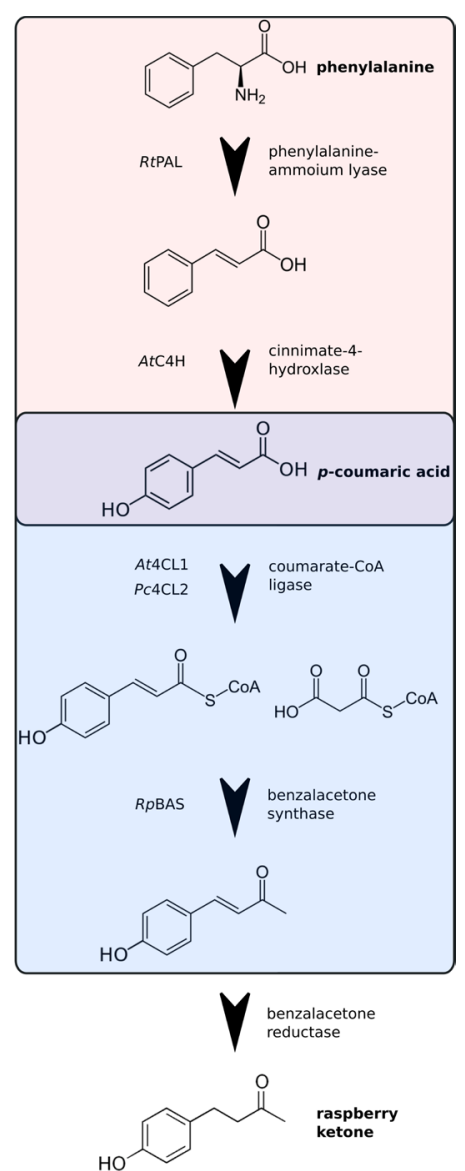

b
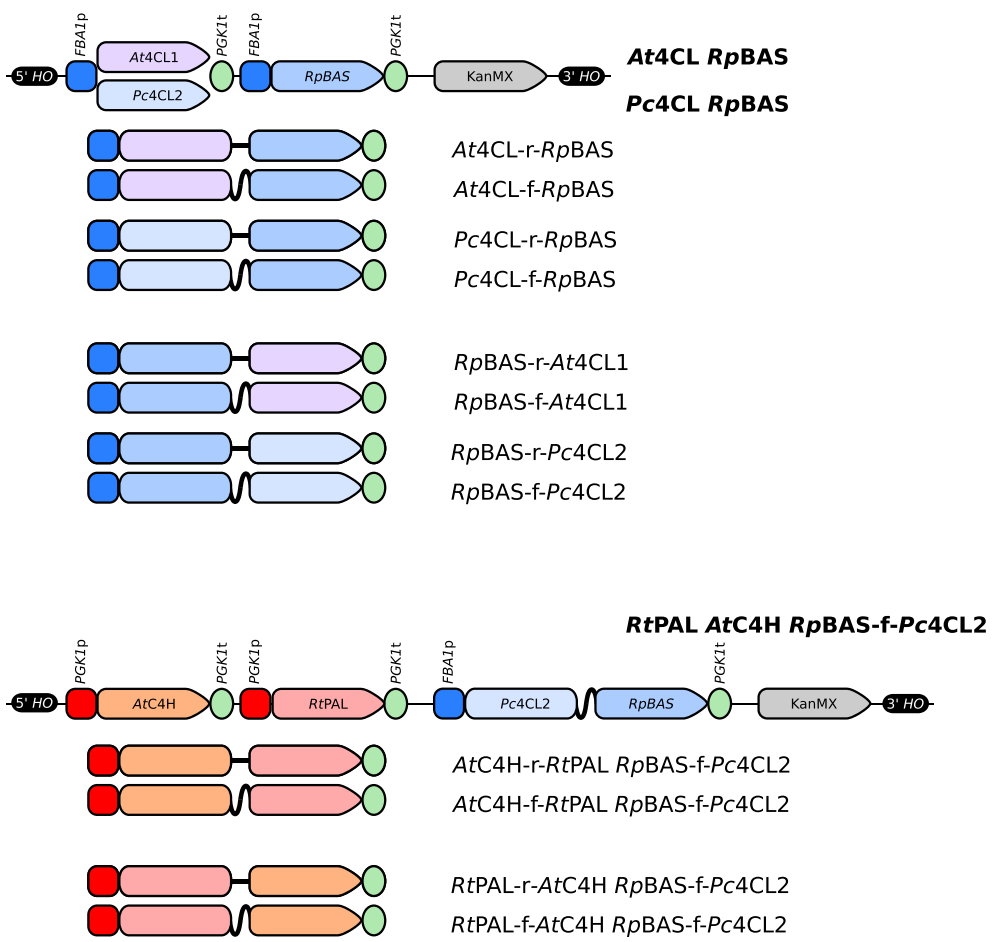

RTPAL-r-AtC4H RpBAS-f-PC4CL2 RTPAL-f-AtC4H RpBAS-f-PC4CL2

Fig. 1 Engineering the raspberry ketone biosynthetic pathway in S. cerevisiae. a The phenylpropanoid pathway begins with the conversion of phenylalanine to $\mathrm{p}$-coumaric acid via cinnamate or directly from tyrosine to $\mathrm{p}$-coumaric acid (pink box). Conversion of $\mathrm{p}$-coumaric acid to raspberry ketone requires three additional enzymatic steps including a condensation reaction between coumaroyl-CoA and malonyl-CoA. Heterologous production of raspberry ketone can be accomplished by the final three enzymatic reactions, if microbial cells are supplied with exogenous $p$-coumaric acid (b/ue box). The heterologous enzymes used for each reaction in this study are also listed. b Expression constructs used in this study for the production of raspberry ketone. Rigid and flexible linker sequences that were used for the protein fusions are represented by bold black lines (straight and wavy, respectively)

lyase (TAL) and cinnamate-4-hydroxylase $(\mathrm{C} 4 \mathrm{H})$, with some enzymes, such as PAL from Rhodosporidium toruloides providing both PAL and TAL activities [17]. The Rhodosporidium toruloides PAL (RtPAL, GenBank: CAD23831.1) and Arabidopsis thaliana C4H (AtC4H, GenBank: AEC08397.1) were therefore chosen as these two enzymes had been shown to function in S. cerevisiae to produce p-coumaric acid previously $[17,18]$.

The RtPAL and AtC4H enzymes were therefore codon optimized and assembled as a binary gene system under the control of the highly-fermentation expressed S. cerevisiae PGK1 promoter [11]. These two genes were then integrated into $H O$ locus of $S$. cerevisiae in combination with the Pc4Cl2-r-RpBAS fusion gene (Fig. 1b).
In anaerobic ferments, the binary gene pair produced $0.49 \pm 0.01 \mathrm{mg} / \mathrm{L}$ of raspberry ketone, representing $18 \%$ of the yield of the Pc4Cl2-r-RpBAS strain supplemented with p-coumaric acid $(2.81 \pm 1.38 \mathrm{mg} / \mathrm{L})$ (Fig. $4 \mathrm{a})$. As performed for the Pc4CL2-RpBAS system, protein fusions were explored using combinations of $A t C 4 \mathrm{H}, R t \mathrm{PAL}$ and either the flexible or rigid linkers to determine if this could result in higher de novo production levels (Fig. 1b). As seen in the Pc4CL2-RpBAS fusions, no significant difference was observed between the use of the rigid and flexible linkers, however unlike the previous system, all four fusions (two linkers $x$ two gene orders) performed significantly worse than the binary gene system (Fig. 4a). Furthermore, the two different fusion orders produced 

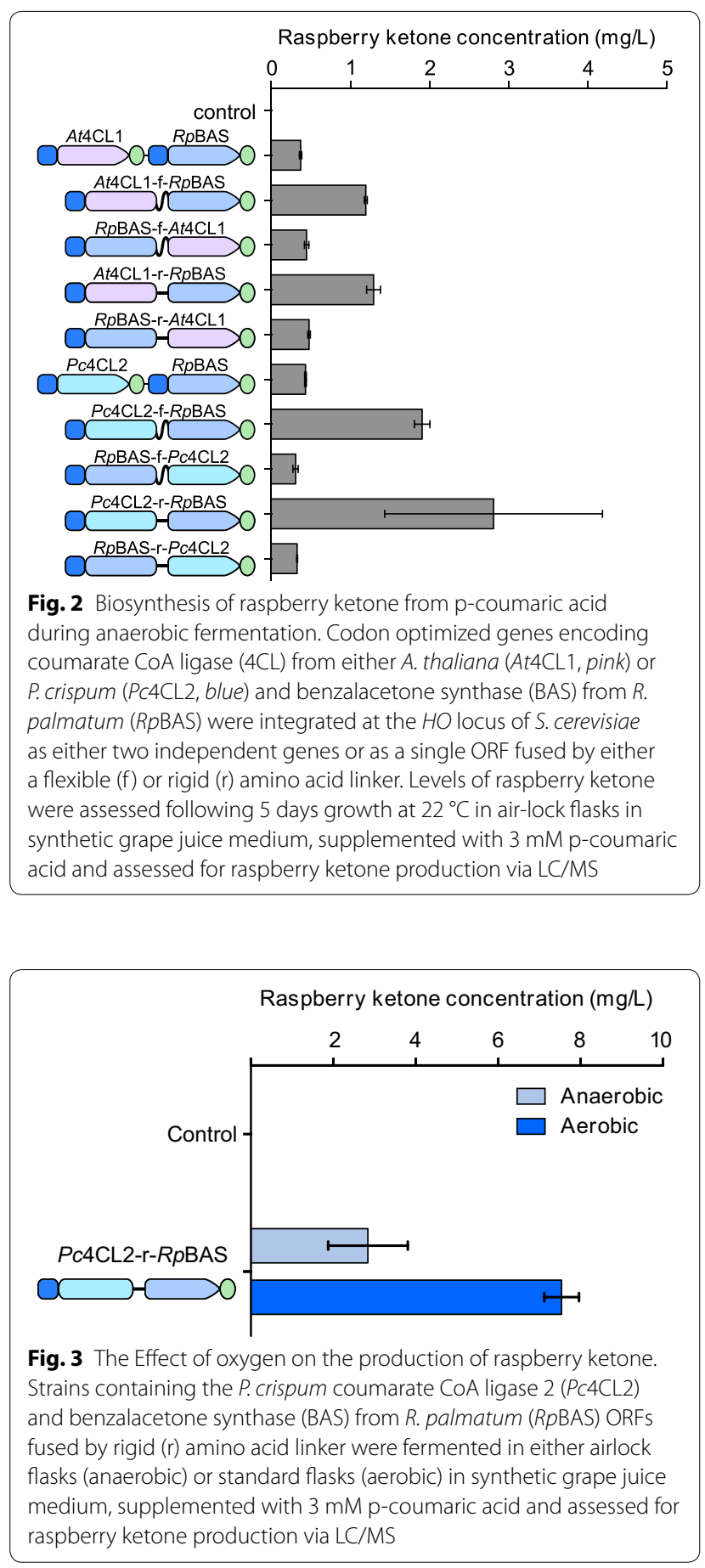

vastly different results, as while the AtC4H-RtPAL fusions reduced activity by $\sim 50 \%(0.28 \pm 0.01 \mathrm{mg} / \mathrm{L})$, the RtPAL-AtC4H fusions almost totally abolished enzyme activity $(0.02-0.04 \mathrm{mg} / \mathrm{L})$.

Having established the de novo production of raspberry ketone under anaerobic conditions, the effect of oxygen was also determined (Fig. 4b). As also observed in

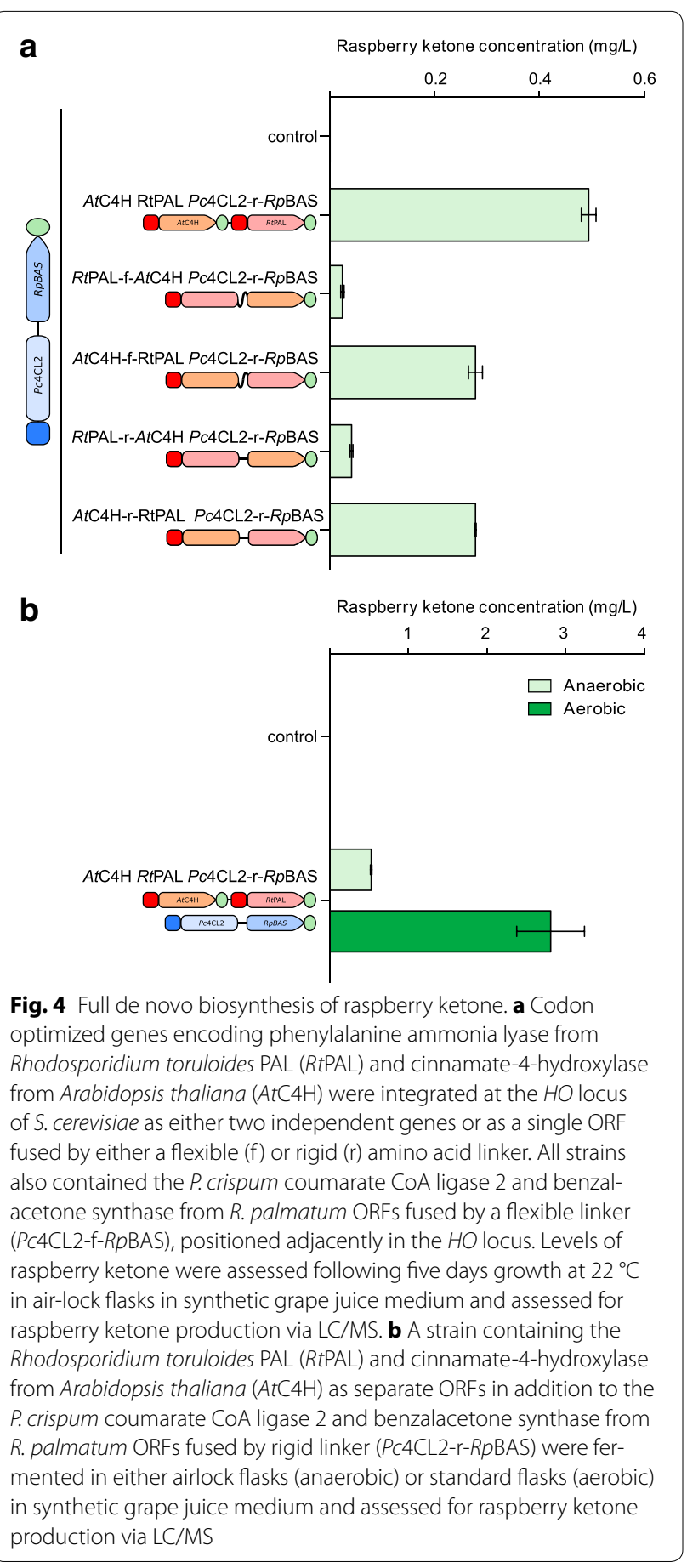

the precursor-fed fermentations, aerobic growth resulted in a significant increase in raspberry ketone levels, to $2.81 \pm 0.43 \mathrm{mg} / \mathrm{L}$ (fivefold).

In order to ensure that the production of raspberry ketone was not impacting cell growth or fermentation ability, the fermentation kinetics of highest raspberry ketone producing strain (AtC4H RtPAL Pc4Cl2-r-RpBAS) 
were compared to both AWRI2975 and AWRI2975 containing the integrated empty vector control under both aerobic and anaerobic growth (Additional file 1: Fig. S1). While the $A t \mathrm{C} 4 \mathrm{H}$ RtPAL Pc4Cl2-r-RpBAS strain displayed a slight delay in total sugar consumption, it was able to finish fermentation efficiently, reaching dryness at the same time point as the parental and control strains.

\section{De novo biosynthesis of raspberry ketone in chardonnay ferments}

Given that a wine strain of $S$. cerevisiae was used for this pathway engineering (AWRI1631) [19], the growth and production of raspberry ketone by the $A t C 4 \mathrm{H} R t \mathrm{PAL}$ $P c 4 C l 2-r-R p$ BAS strain was assessed in Chardonnay grape juice under both aerobic and anaerobic (winemaking) conditions (Fig. 5). There was no significant difference in the levels of raspberry ketone between the synthetic and real grape juice, with $0.68 \pm 0.02$ and $3.49 \pm 0.12 \mathrm{mg} / \mathrm{L}$ of raspberry ketone produced under anaerobic and aerobic conditions, respectively.

\section{Conclusions}

Raspberry ketone represents a highly desirable plantderived compound, providing a major positive aroma in raspberries. The fermentation-derived production of significant amounts of raspberry ketone has been achieved through the introduction of four heterologous genes into S. cerevisiae. This represents the first description of de novo production of raspberry ketone by yeast.

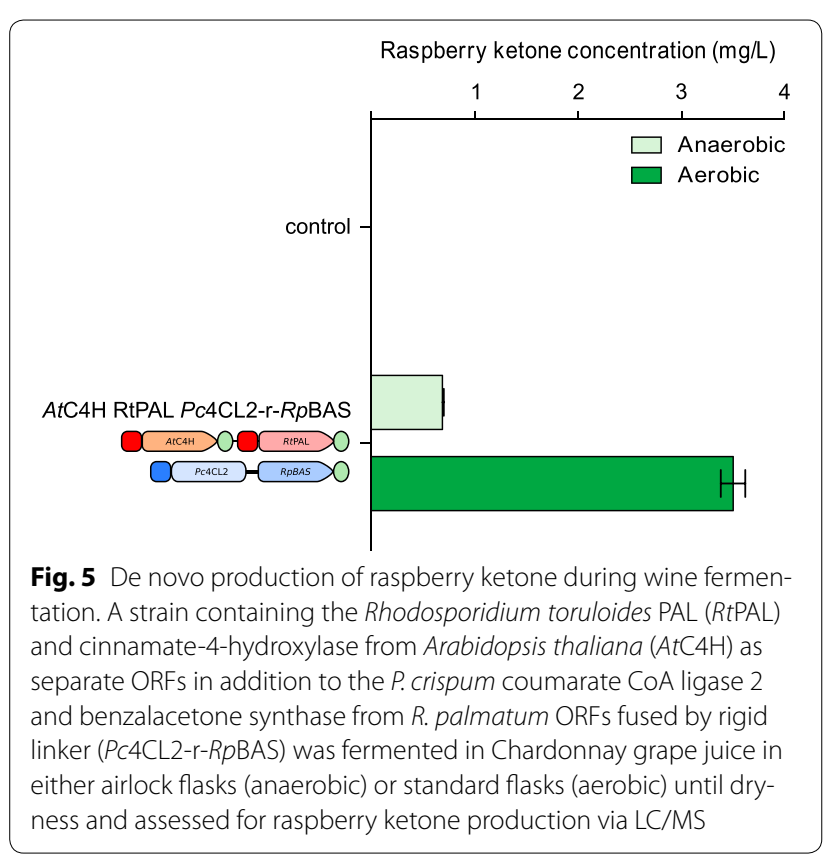

Furthermore, by engineering a synthetic fusion protein between $4 \mathrm{CL}$ and BAS, it was possible to increase the levels of raspberry ketone approximately sixfold, with the maximum de novo production equating to levels generally observed in fresh raspberries. Synthetic fusion proteins have been used extensively in metabolic engineering, where it is thought to allow for increased flux through substrate channeling [20]. In this system, we observed that while proteins were very sensitive to fusion and sometimes, even the particular orientation of the fusion, they were largely insensitive to the predicted secondary structure of the linker (rigid or flexible). This effect was also observed in [16], where the order of farnesyl diphosphate synthase and patchoulol synthase in a protein fusion was shown to be important, but activity was largely insensitive to changes in linker length or secondary structure.

Aerobic culturing consistently resulted in a significant increase in the final concentration of raspberry ketone produced, regardless of the media. Aerobic fermentation would therefore be favored in situations where the maximum amount of raspberry ketone is desired, such as when it is to be isolated as a purified natural flavor product. Purified compounds, such as those produced through the use of engineered microbial strains, are classified as natural flavor compounds and can therefore attract far higher prices than chemically synthesized compounds [4]. Further optimization of raspberry ketone biosynthesis could make the microbial production of purified raspberry ketone economically viable.

The final levels of raspberry ketone produced under winemaking conditions well exceeded the sensory threshold of this compound $(0.001-0.01 \mathrm{mg} / \mathrm{L}$ in water) [2, 14], while the strain was still able to efficiently complete fermentation. While this is a genetically-modified strain and is therefore unavailable for use in most winemaking countries, it would nevertheless be capable of producing a sensorily-augmented product if used in wine fermentation. The production of plant-derived compounds such as raspberry ketone or monoterpenes [21, 22], by engineered yeast highlights the potential for synthetic biology and metabolic engineering to impart novel fermentation properties in industrial strains.

\section{Methods}

\section{ACC1 promoter modification}

To remove acyl-CoA feedback inhibition on the ACC1 gene during fatty acid biosynthesis, the strain AWRI2975 was produced by inserting the constitutive promoter of the $S$. cerevisiae $T D H 3$ gene immediately upstream of the ACC1 start codon in the haploid wine strain AWRI1631 [19] using the delitto perfetto method [23, 24]. 


\section{Gene synthesis}

Rhodosporidium toruloides PAL (RtPAL, GenBank: CAD23831.1), Arabidopsis thaliana C4H (AtC4H, GenBank: AEC08397.1) and 4CL1 (At4CL1, GenBank: AAA82888.1), Petroselinum crispum 4CL2 (Pc4CL2, GenBank: CAA31697.1), and Rheum palmatum BAS (RpBAS, GenBank: AAK82824.1) carrying S338 V mutation were selected to reconstruct the raspberry ketone biosynthesis pathway [9]. Predicted protein sequences of each gene were obtained from GenBank, converted into nucleotide sequences and codon optimized for expression in S. cerevisiae (GeneArt). A NotI restriction site was added before the start codon of each gene, a SalI site was placed immediately in front of the stop codon, and a BamHI site was added after the stop codon to facilitate cloning and the construction of fusion enzymes.

\section{Cloning of raspberry ketone pathway and pathway integration}

Individual synthetic genes were cloned into pCV2-BB entry plasmid, which is a non-replicating variant of pCV3 [25] using the flanking NotI and BamHI sites. Two gene and four gene daisy chains were created by ligating EcoRI-XbaI digested inserts into EcoRI- SpeI digested vectors. Fusion genes were created by ligating hybridized flexible (VDGGSGR) or rigid (VDEAAAKSGR) linker oligonucleotides [16] carrying $5^{\prime}$ SalI and $3^{\prime}$ NotI overhangs with EcoRI-SalI digested pCV2-BB plasmids containing the $5^{\prime}$ genes and EcoRI-NotI digested plasmids containing the 3 ' genes.

The integrating plasmid pCV2-BB-HO1 was created by Gibson assembly [26] through the addition of two $150 \mathrm{bp}$ flanking segments homologous to the $S$. cerevisiae $H O$ locus with $\mathrm{XhoI}$ sites incorporated at the termini. Raspberry ketone pathways constructed in $\mathrm{pCV} 2-\mathrm{BB}$ entry plasmids were sub-cloned into $\mathrm{pCV} 2-\mathrm{BB}-\mathrm{HO} 1$ plasmids, which were digested with $\mathrm{XhoI}$ before being used in yeast transformation.

\section{Culture condition and fermentation}

All yeast strains were maintained on YPD agar plates containing $200 \mathrm{mg} / \mathrm{L}$ G418. Overnight cultures of the yeast strains were grown in $2 \mathrm{~mL}$ YPD containing $200 \mathrm{mg} / \mathrm{L}$ G418 at $28{ }^{\circ} \mathrm{C}$. The overnight YPD cultures were inocu-

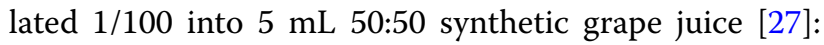
YPD containing $200 \mathrm{mg} / \mathrm{L} \mathrm{G} 418$ and were grown at $28^{\circ} \mathrm{C}$ for $24 \mathrm{~h}$ to an optical density (OD) of $2.60 \pm 0.05$. The overnight synthetic grape juice-YPD cultures were then used as 1:100 starter cultures for ferments. Anaerobic fermentation of synthetic grape juice $(100 \mathrm{~mL})$ were performed in triplicate in $200 \mathrm{~mL}$ Erlenmeyer flasks fitted with water-filled airlocks, incubated at $22{ }^{\circ} \mathrm{C}$ with shaking at $130 \mathrm{rpm}$; aerobic fermentations were performed in triplicate in $200 \mathrm{~mL}$ Erlenmeyer flasks covered with aluminium foil.

For 4CL-BAS strains, p-coumaric acid was added to synthetic grape juice ferments $24 \mathrm{~h}$ post-inoculation to a final concentration of $3 \mathrm{mM}$. Ferments were allowed to proceed for 5 days with the sugar concentrations analysed by HPLC. Chardonnay ferments were performed similarly to synthetic grape juice experiments, but without G418 and were allowed to proceed for 8 days.

\section{LC-MS/MS analysis}

Following cold settling of the ferments, the supernatant for each sample was diluted 1 in 20 with Milli-Q water and analyzed by LC-MS/MS. Calibrants were prepared in matrix (diluted 1 in 20 with Milli-Q water) at levels 0 , $0.5,1,2,5,10,25$ and $50 \mu \mathrm{g} / \mathrm{L}$ from a stock solution of raspberry ketone standard (Sigma Aldrich).

An Agilent 1290 Infinity UHPLC coupled with the 6490 QQQ LC-MS with iFunnel technology was used for the quantification of raspberry ketone. Data acquisition and processing was performed using Mass Hunter software version B.06.00 (Agilent, USA). Samples $(10 \mu \mathrm{L})$ were injected onto a Zorbax Eclipse XDB-C18 Rapid Resolution HT $4.6 \mathrm{~mm} \times 50 \mathrm{~mm} 1.8 \mu \mathrm{m}$ (Agilent, USA). The column temperature was $25^{\circ} \mathrm{C}$. The HPLC mobile phases were $0.1 \%$ formic acid $(\mathrm{v} / \mathrm{v})$ with $10 \mathrm{mM}$ ammonium formate in water (w/v) (solvent A) and methanol (solvent B). An isocratic gradient was used comprising of $50 \%$ solvent $\mathrm{A} /$ solvent $\mathrm{B}$ with a flow rate of $0.6 \mathrm{~mL} / \mathrm{min}$ and a runtime of $5 \mathrm{~min}$. Quantitative analysis was performed using multiple reaction monitoring and ESI positive ionization mode. The following transitions were monitored: $\mathrm{m} / \mathrm{z} 107 \rightarrow 77$ (quantifier), $165 \rightarrow 107$ (qualifier) for raspberry ketone. The following source conditions were used: gas temperature $290{ }^{\circ} \mathrm{C}$; gas flow $14 \mathrm{~L} / \mathrm{min}$; nebulizer $40 \mathrm{psi}$; sheath gas temperature $300{ }^{\circ} \mathrm{C}$; and sheath gas flow $11 \mathrm{~L} / \mathrm{min}$. Nitrogen was used as the nebulizer and collision cell gas. The linear calibration range (LCR) was from 0.5 to $50 \mu \mathrm{g} / \mathrm{L}$ in matrix-matched standards. The limit of detection (LOD) was $0.5 \mu \mathrm{g} / \mathrm{L}$ and the limit of quantification was $1 \mu \mathrm{g} / \mathrm{L}$.

\section{Additional file}

Additional file 1: Fig. S1. Fermentation kenetics of during raspberry ketone production. AWRI2975, AWRI2975 containing an empty vector integrated at $\mathrm{HO}$ (control) and AtC4H RtPAL Pc4Cl2-r-RpBAS (RK) were all used to ferment synthetic Chardonnay juice under either anaerobic ( $A$ ) or aerobic conditions $(\mathrm{B})$. Absorbance $\left(\mathrm{OD}_{600}\right)$ and residual sugar were both recorded at $24 \mathrm{~h}$ intervals for 5 days.

\section{Abbreviations}

4CL: coumarate-CoA ligase; BAS: benzalacetone synthase; BAR: benzalacetone reductase; PAL: phenylalanine ammonia lyase; TAL: tyrosine ammonia lyase; C4H: cinnamate-4-hydroxylase. 


\section{Authors' contributions}

$A R B$ and DL coordinated the study and designed the experiments with input from I.S.P; DL performed the molecular biology and fermentation work; NDRL developed the raspberry ketone assay and coordinated the analysis. All authors contributed to the writing and editing of the manuscript. All authors read and approved the final manuscript.

\section{Author details}

${ }^{1}$ The Australian Wine Research Institute, PO Box 197, Adelaide, SA 5064 , Australia. ${ }^{2}$ Macquarie University, Sydney, NSW 2109, Australia. ${ }^{3}$ Department of Genetics and Evolution, University of Adelaide, Adelaide, SA 5000, Australia.

\section{Acknowledgements}

The Australian Wine Research Institute is a member of the Wine Innovation Cluster in Adelaide. The Synthetic Biology initiative at Macquarie University is financially supported by an internal grant from the University, and external grants from BioPlatforms Australia, the New South Wales (NSW) Chief Scientist and Engineer, and the NSW Government's Department of Primary Industries.

\section{Competing interests}

The authors declare that they have no competing interests.

Received: 15 December 2015 Accepted: 23 February 2016

Published online: 04 March 2016

\section{References}

1. Marienhagen J, Bott M. Metabolic engineering of microorganisms for the synthesis of plant natural products. J Biotechnol. 2013;163:166-78.

2. Larsen M, Poll L. Odour thresholds of some important aroma compounds in raspberries. Z Für Lebensm-Unters Forsch. 1990;191:129-31.

3. Larsen M, Poll L, Callesen O, Lewis M. Relations between the content of aroma compounds and the sensory evaluation of 10 raspberry varieties (Rubus idaeus L). Acta Agric Scand. 1991;41:447-54.

4. Stabnikova O, Wang J-Y, Ivanov V. Value-added biotechnological products from organic wastes. In: Wang LK, Ivanov V, Tay J-H. Totowa, NJ, editors. Environmental biotechnology. Humana Press; 2010: 343-394.

5. Beekwilder J, van der Meer IM, Sibbesen O, Broekgaarden M, Qvist I, Mikkelsen JD, Hall RD. Microbial production of natural raspberry ketone. Biotechnol J. 2007;2:1270-9.

6. Yan Y, Huang L, Koffas MAG. Biosynthesis of 5-deoxyflavanones in microorganisms. Biotechnol J. 2007;2:1250-62.

7. Sydor T, Schaffer S, Boles E. Considerable increase in resveratrol production by recombinant industrial yeast strains with use of rich medium. Appl Environ Microbiol. 2010;76:3361-3.

8. Abe I, Takahashi Y, Morita H, Noguchi H. Benzalacetone synthase. A novel polyketide synthase that plays a crucial role in the biosynthesis of phenylbutanones in Rheum palmatum. Eur J Biochem. 2001;268:3354-9.

9. Abe T, Morita H, Noma H, Kohno T, Noguchi H, Abe I. Structure function analysis of benzalacetone synthase from Rheum palmatum. Bioorg Med Chem Lett. 2007;17:3161-6.

10. Varela C, Cárdenas J, Melo F, Agosin E. Quantitative analysis of wine yeast gene expression profiles under winemaking conditions. Yeast. 2005;22:369-83

11. Varela C, Kutyna DR, Solomon MR, Black CA, Borneman A, Henschke PA, Pretorius IS, Chambers PJ. Evaluation of gene modification strategies for the development of low-alcohol-wine yeasts. Appl Environ Microbiol. 2012;78:6068-77.

12. Baganz F, Hayes A, Marren D, Gardner DC, Oliver SG. Suitability of replacement markers for functional analysis studies in Saccharomyces cerevisiae. Yeast. 1997;13:1563-73.
13. Voth WP, Richards JD, Shaw JM, Stillman DJ. Yeast vectors for integration at the HO locus. Nucleic Acids Res. 2001;29:e59.

14. Ohloff G. Recent developments in the field of naturally-occurring aroma components: progress in the chemistry of organic natural products. In: Herz W, Grisebach H, Kirby GW, editors. Progress in the chemistry of organic natural products. Vienna: Springer; 1978. vol. 35 p. 431-527.

15. Lu P, Feng M-G. Bifunctional enhancement of a beta-glucanase-xylanase fusion enzyme by optimization of peptide linkers. Appl Microbiol Biotechnol. 2008;79:579-87.

16. Albertsen L, Chen Y, Bach LS, Rattleff S, Maury J, Brix S, Nielsen J, Mortensen UH. Diversion of flux toward sesquiterpene production in Saccharomyces cerevisiae by fusion of host and heterologous enzymes. Appl Environ Microbiol. 2011;77:1033-40.

17. Vannelli T, Wei Qi W, Sweigard J, Gatenby AA, Sariaslani FS. Production of p-hydroxycinnamic acid from glucose in Saccharomyces cerevisiae and Escherichia coli by expression of heterologous genes from plants and fungi. Metab Eng. 2007;9:142-51.

18. Shin S-Y, Jung S-M, Kim M-D, Han NS, Seo J-H. Production of resveratrol from tyrosine in metabolically engineered Saccharomyces cerevisiae. Enzyme Microb Technol. 2012;51:211-6.

19. Borneman AR, Forgan AH, Pretorius IS, Chambers PJ. Comparative genome analysis of a Saccharomyces cerevisiae wine strain. FEMS Yeast Res. 2008;8:1185-95.

20. Conrado RJ, Varner JD, DeLisa MP. Engineering the spatial organization of metabolic enzymes: mimicking nature's synergy. Curr Opin Biotechnol. 2008;19:492-9.

21. Herrero O, Ramón D, Orejas M. Engineering the Saccharomyces cerevisiae isoprenoid pathway for de novo production of aromatic monoterpenes in wine. Metab Eng. 2008;10:78-86.

22. Pardo E, Rico J, Gil JV, Orejas M. De novo production of six key grape aroma monoterpenes by a geraniol synthase-engineered $\mathrm{S}$. cerevisiae wine strain. Microb Cell Factories. 2015;14:136.

23. Storici F, Resnick MA. The delitto perfetto approach to in vivo sitedirected mutagenesis and chromosome rearrangements with synthetic oligonucleotides in yeast. Methods Enzymol. 2006:409:329-45.

24. Wattanachaisaereekul S, Lantz AE, Nielsen ML, Nielsen J. Production of the polyketide 6-MSA in yeast engineered for increased malonyl-CoA supply. Metab Eng. 2008;10:246-54.

25. Krömer JO, Nunez-Bernal D, Averesch NJH, Hampe J, Varela J, Varela C. Production of aromatics in Saccharomyces cerevisiae - a feasibility study. J Biotechnol. 2013;163:184-93.

26. Gibson DG, Young L, Chuang R-Y, Venter JC, Hutchison CA, Smith HO. Enzymatic assembly of DNA molecules up to several hundred kilobases. Nat Methods. 2009;6:343-5.

27. Schmidt SA, Dillon S, Kolouchova R, Henschke PA, Chambers PJ. Impacts of variations in elemental nutrient concentration of Chardonnay musts on Saccharomyces cerevisiae fermentation kinetics and wine composition. Appl Microbiol Biotechnol. 2011;91:365-75.

\section{Submit your next manuscript to BioMed Central and we will help you at every step:}

- We accept pre-submission inquiries

- Our selector tool helps you to find the most relevant journal

- We provide round the clock customer support

- Convenient online submission

- Thorough peer review

- Inclusion in PubMed and all major indexing services

- Maximum visibility for your research

Submit your manuscript at www.biomedcentral.com/submit

() Biomed Central 